\title{
Update on the Preventive Antibiotics in Stroke Study (PASS): a randomised controlled phase 3 clinical trial
}

Willeke F Westendorp ${ }^{1 \dagger}$, Jan-Dirk Vermeij ${ }^{1 \dagger}$, Nan van Geloven ${ }^{2}$, Diederik WJ Dippel ${ }^{3}$, Marcel GW Dijkgraaf², Tom van der Poll ${ }^{4,5}$, Jan M Prins ${ }^{4,5}$, Lodewijk Spanjaard ${ }^{6}$, Frederique H Vermeij ${ }^{7}$, Paul J Nederkoorn ${ }^{1,8^{*}+}$ and Diederik van de Beek ${ }^{1,4 \dagger}$

\begin{abstract}
Background: Stroke is a leading cause of death worldwide. Infections after stroke occur in 30\% of stroke patients and are strongly associated with unfavourable outcome. Preventive antibiotic therapy lowers infection rate in patients after stroke, however, the effect of preventive antibiotic treatment on functional outcome after stroke has not yet been investigated.The Preventive Antibiotics in Stroke Study (PASS) is an ongoing, multicentre, prospective, randomised, open-label, blinded end point trial of preventive antibiotic therapy in acute stroke. Patients are randomly assigned to either ceftriaxone at a dose of $2 \mathrm{~g}$, given every 24 hours intravenously for four-days, in addition to stroke-unit care, or standard stroke-unit care without preventive antibiotic therapy. Aim of the study is to assess whether preventive antibiotic treatment improves functional outcome at three months by preventing infections.

Results: To date, 2,470 patients have been included in PASS. Median stroke severity of the first 2,133 patients (second interim analysis) is 5 (IQR 3 to 9) on the National Institutes of Health Stroke Scale (NIHSS). Due to the PROBE design, no outcome data are available yet. In the initial trial protocol we proposed a dichotomisation of the mRS as primary analysis of outcome and ordinal regression analysis as secondary analysis of primary outcome, requiring a sample size of 3,200 patients. However, ordinal analysis of outcome data is becoming increasingly more common in acute stroke trials, as it increases statistical power. For PASS, funding is insufficient for inclusion of 3,200 patients with the overall inclusion rate of 15 patients per week. Therefore we change the analysis of our primary outcome from dichotomisation to ordinal regression analysis on the mRS. Power analysis showed that with similar assumptions 2,550 patients are needed using ordinal regression analysis. We expect to complete follow-up in June 2014. A full statistical analysis plan will be submitted for publication before treatment allocation will be unblinded.
\end{abstract}

Conclusion: The data from PASS will establish whether preventive antibiotic therapy in acute stroke improves functional outcome by preventing infection. In this update, we changed our primary outcome analysis from dichotomisation to ordinal regression analysis.

Trial registration: Current controlled trials; ISRCTN66140176. Date of registration: 6 April 2010.

Keywords: Stroke, Infection, Antibiotics

\footnotetext{
* Correspondence: p.j.nederkoorn@amc.uva.nl

${ }^{\dagger}$ Equal contributors

'Department of Neurology, Academic Medical Centre, Amsterdam, The

Netherlands

${ }^{8}$ Department of Neurology, H2.216, Academic Medical Centre, PO box 22660,

Amsterdam 1100 DD, The Netherlands

Full list of author information is available at the end of the article
}

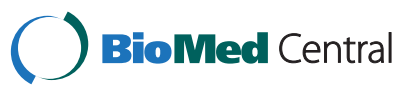

(c) 2014 Westendorp et al.; licensee BioMed Central Ltd. This is an Open Access article distributed under the terms of the Creative Commons Attribution License (http://creativecommons.org/licenses/by/4.0), which permits unrestricted use, distribution, and reproduction in any medium, provided the original work is properly credited. The Creative Commons Public Domain Dedication waiver (http://creativecommons.org/publicdomain/zero/1.0/) applies to the data made available in this article, unless otherwise stated. 


\section{Update}

\section{Preventive Antibiotics in Stroke Study (PASS)}

Stroke is a leading cause of death worldwide. Infections after stroke occur in $30 \%$ of stroke patients and are strongly associated with unfavourable outcome $[1,2]$. Preventive antibiotic therapy lowers infection rate in patients after stroke; however, the effect of preventive antibiotic treatment on functional outcome after stroke has not yet been investigated $[3,4]$.

The aim of PASS is to investigate whether preventive use of the antibiotic ceftriaxone improves functional outcome in patients with stroke. PASS is an ongoing, multicentre Prospective, Randomised, Open-label, Blinded End point trial (PROBE) of standard care with preventive ceftriaxone treatment which is compared with standard care without preventive ceftriaxone. Adult patients with stroke (both ischaemic and haemorrhagic) and a score $\geq 1$ on the National Institutes of Health Stroke Scale will be included. Patients are randomly assigned to either ceftriaxone at a dose of $2 \mathrm{~g}$, given every 24 hours intravenously for four-days, in addition to stroke-unit care, or standard stroke-unit care without preventive antibiotic therapy. All items from the World Health Organization Trial Registration Data Set are shown in Table 1. For description of the entire study protocol, including study procedures and data collection, assessment of infections and outcomes, allocation and blinding procedures, we refer to the initial trial protocol publication [5]. Changes to the protocol since the first version are shown in Table 2. Medical-ethical approval of the protocol and amendments was obtained by the medical ethical committee of the AMC. All participating centres are shown in Table 3.

The primary end point of the PASS is functional outcome at three-month follow-up on the modified Rankin Scale (mRS), a well-validated functional outcome scale in stroke patients [6]. In the protocol publication, the primary efficacy end point has been defined as the functional outcome at the three-month follow-up, as assessed by the mRS dichotomised as a favourable outcome ( $\mathrm{mRS} 0$ to 2 ) or as an unfavourable outcome (mRS 3 to 6 ). The proportional odds model was defined as the secondary analysis of the primary end point [5]. Secondary outcome measures were death rate at discharge and three-months, infection rate during hospital admission, length of hospital admission, volume of post-stroke care, use of antibiotics during follow-up, Quality-adjusted life years (QALYs); and costs. In this update publication of PASS, we change our primary outcome analysis from dichotomisation to ordinal regression analysis on the mRS. We also change the secondary outcome of use of antibiotics during follow-up into use of antibiotics during hospital stay.

\section{Change in primary analysis of primary outcome and adaptation of sample size}

The modified Rankin Scale is a well-validated functional scale for assessing outcome after stroke. Analysis on a dichotomisation in favourable versus unfavourable outcome delivers easily comprehensible results. However, cut-off is arbitrarily and solely based on improvement beyond this one cut-off point. A secondary analysis including 55 datasets of stroke trials showed that statistical analysis based on the ordered nature of functional outcome data versus dichotomisation was more efficient and more likely to deliver reliable results [7]. Although there were some annotations regarding this publication, more and more studies are using ordinal regression analysis [8-10].

In the design of PASS, both dichotomisation and ordinal regression analysis were described as analysis of the primary outcome [5]. We based our initial sample size calculation on the dichotomised outcome (favourable versus unfavourable outcome). Dichotomisation was chosen as primary analysis of efficacy because of the widespread use in stroke trials $[5,11]$. However, trial completion will take an unrealistically long time with excessive costs with the current inclusion rate of 15 patients per week. Therefore, we now propose a switch in primary analysis of the primary outcome using an ordinal outcome analysis. The primary outcome will remain to be assessed on the mRS. The primary outcome with dichotomisation will be presented as secondary analysis of primary outcome. Using ordinal regression analysis for PASS enables us to preserve the assumptions of the strength of the treatment effect with a lower total sample size.

\section{Sample size}

We based our initial sample size calculation on the dichotomised outcome (favourable versus unfavourable outcome). With the assumption of reduction of unfavourable outcome of $5 \%$, with a power of $80 \%$ and $P$-value of 0.05 , we aimed to include 3,200 patients.

We now propose a new sample size of 2,550 patients, which is based on the ordinal regression analysis of the primary outcome. For this analysis we will use the 'proportional odds model', also known as the 'cumulative logit model' [12]. The assumption for the distribution on $\mathrm{mRS}$ in the control-arm is based on the control-arm in the Paracetamol (Acetaminophen) In Stroke (PAIS) trial, which had almost similar inclusion criteria as PASS [13]. We assumed a proportional odds ratio of 0.818 between all pairs of category groups, similar to the assumption in the original sample size calculation (odds ratio of 0.818 for mRS 0 to 2 versus mRS 3 to 6). Figure 1 shows the expected distribution of the two treatment arms. Using the method of Whitehead, with alpha 0.05 and power 
Table 1 All items from the World Health Organization Trial Registration Data Set (SPIRIT checklist, item 2b)

\section{Data category}

Primary registry and trial

identifying number

Date of registration in

primary registry

Secondary identifying numbers

Source(s) of monetary or

material support

Primary sponsor

Secondary sponsor(s)

Contact for public queries

Contact for scientific queries

Public title

Scientific title

Countries of recruitment

Health condition(s) or problem(s) studied

Intervention(s)

Key inclusion and exclusion criteria

Study type

Date of first enrolment

Target sample size

Recruitment status

Primary outcome(s)

Key secondary outcomes

\section{Information}

Current controlled trials; www.controlled-trials.com; ISRCTN66140176

6 April 2010

1. Netherlands Organisation for Health Research and Development (ZonMw) (Netherlands) (ref: 171002302) 2. Netherlands Heart Foundation (Nederlandse Hartstichting) (Netherlands) (ref: CD 300006)

Academic Medical Centre (AMC) (Netherlands)

Paul J Nederkoorn; P.J.Nederkoorn@amc.uva.nl

Paul J Nederkoorn, Department of Neurology, Academic Medical Centre, PO box 22660, 1100 DD Amsterdam, The Netherlands.

Preventive Antibiotics in Stroke Study

Preventive ceftriaxone to improve functional health in patients with stroke by preventing infection: a multicentre prospective randomised controlled trial

The Netherlands

Stroke, infection

Optimal medical care and ceftriaxone 2,000 mg intravenously, once daily, for four days, versus optimal medical care without ceftriaxone.

Inclusion criteria: aged greater than or equal to 18 years, either sex; stroke (ischaemic and haemorrhagic); any measurable neurological deficit defined as National Institutes of Health Stroke Scale (NIHSS) greater than 1; stroke onset less than 24 hours; admission.

Exclusion criteria: symptoms or signs of infection on admission requiring antibiotic therapy; use of antibiotics less than 24 hours before admission; pregnancy; hypersensitivity for cephalosporin; previous anaphylaxis for penicillin or derivates; subarachnoid haemorrhage; death seems imminent.

Multicentre prospective randomised open-label blinded end point trial

4 July 2010

2,550

Recruiting

Functional health at three-month follow-up, as assessed by the modified Rankin Scale (mRS)

Death rate at discharge and three months, infection rate during hospital admission; length of hospital admission; volume of post-stroke care; use of antibiotics during hospital stay; Quality adjusted life years (QALYS); costs.

Table 2 Protocol revision chronology

\begin{tabular}{ll}
\hline Date & Protocol version and amendments \\
\hline 5 May 2010 & Original protocol \\
& Protocol version 1.1. Amendments: exclusion criterion 'death seems imminent' added; compulsory urine analysis and \\
& culture on admission omitted. \\
& Protocol version 1.2. Amendments: new study centres with new estimations of included patients were added; paragraph 6.6 \\
& 'drug-accountability': badge number of the administered ceftriaxone will be noted by the nurse administrating the medication \\
& into the 'drug accountability form' according to GCP-guidelines for pharmacies; paragraph 7.2 'randomisation, blinding and \\
& treatment allocation': randomisation will not be stratified according to stroke type, solely by study centre and stroke severity; \\
& assessment of blinded outcome is specified as performed by a person not involved in the trial team; performance of interim \\
& analyses is specified as performed by an independent statistician not involved in the trial team; paragraph 8.2 'adverse and \\
& serious adverse events': for each participating centre, a flowchart of serious adverse event/suspected unexpected serious \\
& adverse reactions (SAE/SUSAR) reporting will be provided in the local Investigator File; paragraph 8.5 'data monitoring': \\
& reference to the monitoring plan is added.
\end{tabular}

10 January $2014 \quad$ Protocol version 1.3. Amendment: change in primary analysis of primary outcome from dichotomised analysis to ordinal regression analysis according to the proportional odds model.

Total course of study Participating centres were added (all participating centres are shown in Table 3). 
Table 3 Centres participating in the Preventive Antibiotics in Stroke Study (PASS) with local investigators

\begin{tabular}{|c|c|}
\hline Centre & Local investigator \\
\hline Academic Medical Centre, Amsterdam & PJ Nederkoorn; D van de Beek \\
\hline Albert Schweitzer Hospital, Dordrecht & H Kerkhoff \\
\hline Amphia Hospital, Breda & MJM Remmers \\
\hline Amstelland Hospital, Amstelveen & DSM Molenaar \\
\hline Atrium Medical Centre, Heerlen & T Schreuder \\
\hline Boven-IJ Hospital, Amsterdam & M Janmaat \\
\hline Bronovo Hospital, The Hague & SM Manschot \\
\hline Catharina Hospital, Eindhoven & K Keizer \\
\hline Erasmus Medical Centre, Rotterdam & DWJ Dippel \\
\hline Groene Hart Hospital, Gouda & K de Gans \\
\hline HAGA Hospital, The Hague & SF de Bruijn \\
\hline Kennemer Gasthuis, Haarlem & M Weisfelt \\
\hline Laurentius Hospital, Roermond & ML van Goor \\
\hline Martini Hospital, Groningen & ES Schut \\
\hline Medical Centre Haaglanden, The Hague & K Jellema \\
\hline Medical Centre Alkmaar & R ten Houten \\
\hline $\begin{array}{l}\text { Onze Lieve Vrouwe } \\
\text { Gasthuis Amsterdam }\end{array}$ & JLM Bosboom \\
\hline Orbis Medical Centre, Sittard & $\mathrm{N}$ van Orshoven \\
\hline Rijnstate Hospital, Arnhem & SE Vermeer \\
\hline Reinier de Graaf Hospital, Delft & LAM Aerden \\
\hline Slotervaart Hospital, Amsterdam & ND Kruyt \\
\hline Spaarne Hospital, Hoofddorp & ISJ Merkies \\
\hline St. Franciscus Gasthuis, Rotterdam & FH Vermeij \\
\hline $\begin{array}{l}\text { University Medical Centre } \\
\text { Radboud, Nijmegen }\end{array}$ & E van Dijk \\
\hline University Medical Centre, Utrecht & HB van der Worp \\
\hline VU Medical Centre, Amsterdam & MC Visser \\
\hline Westfries Gasthuis Hoorn & TC van der Ree \\
\hline ljsselland Hospital, Capelle aan den IJssel & AD Wijnhoud \\
\hline Zaans Medical Centre, Zaandam & RM van den Berg - Vos \\
\hline ZGT, Almelo & LJA Reichman \\
\hline
\end{tabular}

$80 \%$, the desired sample size in the proportional odds model is estimated at a total of 2,410 patients [14]. Given an expected rate of patients lost to follow-up and/or patients with incomplete data of $5 \%$, a conservative estimate for the new sample size with the primary end point analysed on all categories of the mRS is 2,531 patients. We will therefore adapt the sample size to 2,550 patients; a reduction of 650 patients compared to the original sample size estimate based on a dichotomous outcome on the mRS. This decision has been made by the researchers without any knowledge of outcome data per treatment group.

\section{Recruitment target}

By 12 February 2014, 2,470 patients were included in the PASS. Up-to-date statistics can be found at www. passamc.nl. With a stable weekly inclusion rate of 15 patients, follow-up of the last included patient is expected in June 2014.

\section{Definitions of infection}

Infection rate during hospital admission will be assessed in two ways. First, clinical diagnosis according to the treating physician will be recorded. Second, diagnosis of infection will be judged by two experienced infectious diseases specialists, blinded for treatment allocation, using the modified criteria of the United States Centres for Disease Control and Prevention [15]. This will be done in all patients who developed fever or a new onset delirium during admission, in patients in whom there was suspicion of infection but no diagnostics were performed, and in patients in a palliative care setting. One important issue that needs to be addressed is the risk of performance and detection bias. Since the treating physician is aware of the treatment allocation, this could influence decisions on non-scheduled treatment. For the PASS, the most important issue to address is the detection and treatment of infection. A physician could be more or less likely to order investigations or start treatment for a possible infection depending on the treatment allocation. By giving recommendations for diagnostic procedures in the previously mentioned subgroups of patients, and by collecting results of these procedures in standardized case record forms, we try to limit this form of bias.

\section{Monitoring of antibiotic resistance}

One of the most important mechanisms of resistance against third generation cephalosporins is forming of extended-spectrum- $\beta$-lactamase (ESBL), an enzyme that renders antibiotics ineffective, in Enterobacteriaceae. In our study we monitor the prevalence of ESBL-producing bacteria in both treatment arms. We therefore collect stool specimens at admission and discharge in a subgroup of patients. To date, samples have been obtained in 300 patients.

\section{Development of the statistical analysis plan}

Currently, the statistical analysis plan is being finalised, without insight in to the unblinded data. It will be published before the randomisation code is broken in late 2014. The statistical analysis plan describes the analysis of primary outcome with ordinal regression analysis and a secondary dichotomised analysis into detail. It also describes a small number of prespecified subgroup analyses, and a larger number of exploratory secondary analyses, that will be performed, as well as treatment of missing values. 


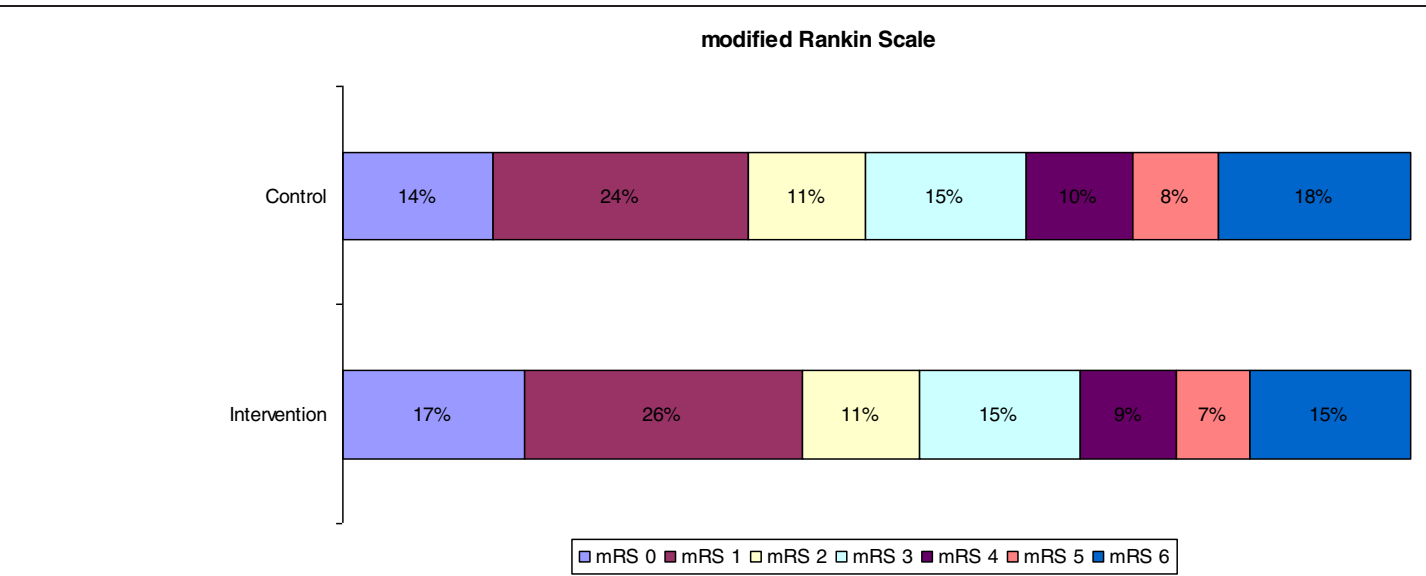

Figure 1 Expected distribution of the two treatment arms. The assumption for the distribution on mRS in the control-arm is based on the control-arm in the Paracetamol (Acetaminophen) In Stroke (PAIS) trial, which had almost similar inclusion criteria as PASS [9].

\section{Discussion}

The PASS aims to investigate whether preventive antibiotic therapy improves functional outcome by preventing infections. The results of a trial examining the effect of preventive antibiotic therapy on functional outcome are urgently warranted. Infection after stroke is common and infection has repeatedly been shown to worsen outcome $[1,2,16,17]$. Since previous studies on preventive antibiotic therapy were too small, heterogeneous, or did not investigate functional outcome, no sufficient information is available on the role of preventive antibiotic therapy in acute stroke [4].

With this update of the protocol, we present a change in primary analysis of the primary outcome on the mRS from a dichotomised analysis to an ordinal regression analysis. Ordinal analysis of outcome data is increasingly common in acute stroke trials as well as in other trials, for example on traumatic brain injury $[7,18]$. Data is used more efficiently with ordinal analysis as compared to a dichotomised analysis. For example the ECASS-II trial failed to show an effect of treatment in the dichotomised approach, but did show an affect with ordinal shift analysis [19].

Different approaches can be used for the analysis of ordinal outcome data. In the PASS we chose the ordinal regression analysis as primary analysis of outcome, which was already described as a secondary analysis of primary outcome in the original protocol. The proportional odds model provides additional information from ordinal outcome data, as it takes into account improvements at any point on the mRS [18]. This method is highly efficient when compared to a dichotomised approach, but also when compared to other ordinal approaches [18]. A possible disadvantage of this approach is the assumption of proportional odds across all groups. In PASS, we chose this method because we expected a similar effect of preventive antibiotic treatment across all outcomes, and therefore expect to meet the assumptions of the proportional odds model.

With the new sample size of 2,550 patients we expect completion of inclusion of patients in PASS in June 2014.

\section{Abbreviations}

AMC: Academic Medical Centre; ESBL: extended-spectrum-ß-lactamase, mRS, modified Rankin Scale; NIHSS: National Institutes of Health Stroke Scale; PASS: Preventive Antibiotics in Stroke Study; PROBE: Prospective, Randomised, Open-label, Blinded End point; SAE: serious adverse event; SUSAR: suspected unexpected serious adverse reaction.

\section{Competing interests}

All authors declare not to have any competing interests.

\section{Authors' contributions}

WFW, JDV performed conception and design of the work and drafted the work. PJN and DvdB performed conception and design of the work and revised it critically for important intellectual content. NG performed the statistical analysis for this paper and revised it critically. DWJD, MGWD, TvdP, JMP, LS, FHV were involved in design of the work, and revised it critically for intellectual content. All authors approved the final version.

\section{Acknowledgements}

The PASS study was funded by the Academic Medical Centre (AMC), by the Netherlands Organisation for Health Research and Development (ZonMW; 171002302) and the Netherlands Heart Foundation (Hartstichting; 2009B095). Principal investigators of the PASS are Dr. PJ Nederkoorn and Professor D van de Beek. DvdB is supported by grants from the European Research Council (ERC Starting Grant (Proposal/Contract number 281156)), Netherlands Organization for Health Research and Development (ZonMw; NWO-Vidi grant 2010 (Proposal/Contract number 016.116.358)). The study group comprises Professor DWJ Dippel; Dr. MGW Dijkgraaf; Professor JM Prins; Dr. L Spanjaard; Professor T van der Poll; Dr. FH Vermeij. Two PhD students working on the PASS are Dr. WF Westendorp and Dr. J-D Vermeij. Trial manager and nurses are Drs. IJ Hooijenga, AG de Jong and I Stijnman- Moerman.

Thirty Dutch hospitals participate in the PASS; all centres with local investigators are shown in Table.

The data safety monitoring committee (DSMB) is formed by: GJ Hankey, MD, PhD, Consultant Neurologist, Head of Stroke Unit, Department of Neurology, Royal Perth Hospital, Australia (chair); A Algra, MD, PhD, Clinical

Epidemiologist, Julius Centre and Department of Neurology, UMC Utrecht, the Netherlands; MJM Bonten, MD, PhD, Microbiologist, Department of Medical Microbiology and Julius Centre, University Medical Centre Utrecht, Utrecht, the Netherlands. Advisory Board of the PASS consists of Professor M Vermeulen, Department of Neurology, AMC Amsterdam, and Professor RJ de 
Haan, Clinical Research Unit, AMC Amsterdam. We like to thank Professor P Sandercock, Department of Clinical Neurosciences, Western General Hospital, Edinburgh, for advising us with the design of the current protocol update.

\section{Author details}

'Department of Neurology, Academic Medical Centre, Amsterdam, The Netherlands. ${ }^{2}$ Clinical Research Unit (CRU), Academic Medical Centre, Amsterdam, The Netherlands. ${ }^{3}$ Department of Neurology, Erasmus MC University Medical Centre, Rotterdam, The Netherlands. ${ }^{4}$ Centre of Infection and Immunity (CINIMA), Academic Medical Centre, Amsterdam, The Netherlands. ${ }^{5}$ Department of Infectious Diseases, Academic Medical Centre, Amsterdam, The Netherlands. ${ }^{6}$ Department of Microbiology, Academic Medical Centre, Amsterdam, The Netherlands. 'Department of Neurology, Sint Franciscus Gasthuis, Rotterdam, The Netherlands. ${ }^{8}$ Department of Neurology, H2.216, Academic Medical Centre, PO box 22660, Amsterdam 1100 DD, The Netherlands.

Received: 24 February 2014 Accepted: 2 April 2014

Published: 21 April 2014

\section{References}

1. Vermeij FH, op Reimer WJ S, de MP, Van Oostenbrugge RJ, Franke CL, de Jong G, de Kort PL, Dippel DW, Netherlands Stroke Survey Investigators: Stroke-associated infection is an independent risk factor for poor outcome after acute ischemic stroke: data from the Netherlands Stroke Survey. Cerebrovasc Dis 2009, 27:465-471.

2. Westendorp WF, Nederkoorn PJ, Vermeij JD, Dijkgraaf MG, van de Beek D: Post-stroke infection: a systematic review and meta-analysis. BMC Neurol 2011, 11:110.

3. van de Beek D, Wijdicks EF, Vermeij FH, de Haan RJ, Prins JM, Spanjaard L, Dippel DW, Nederkoorn PJ: Preventive antibiotics for infections in acute stroke: a systematic review and meta-analysis. Arch Neurol 2009, 66:1076-1081.

4. Westendorp WF, Vermeij JD, Vermeij F, den Hertog HM, Dippel DW, van de Beek D, Nederkoorn PJ: Antibiotic therapy for preventing infections in patients with acute stroke. Cochrane Database Syst Rev 2012, 1:CD008530.

5. Nederkoorn PJ, Westendorp WF, Hooijenga IJ, de Haan RJ, Dippel DW Vermeij FH, Dijkgraaf MG, Prins JM, Spanjaard L, van de Beek D: Preventive antibiotics in stroke study: rationale and protocol for a randomised trial. Int J Stroke 2011, 6:159-163.

6. Banks $J$, Marotta CA: Outcomes validity and reliability of the modified Rankin scale: implications for stroke clinical trials: a literature review and synthesis. Stroke 2007, 38:1091-1096.

7. Bath PM, Gray L, Collier T, Pocock S, Carpenter J: Can we improve the statistical analysis of stroke trials? Statistical reanalysis of functional outcomes in stroke trials. Stroke 2007, 38:1911-1915.

8. Anderson CS, Heeley E, Huang Y, Wang J, Stapf C, Delcourt C, Lindley R, Robinson T, Lavados P, Neal B, Hata J, Arima H, Parsons M, Li Y, Wang J, Heritier S, Li Q, Woodward M, Simes RJ, Davis SM, Chalmers J, INTERACT2 Investigators: Rapid blood-pressure lowering in patients with acute intracerebral hemorrhage. N Engl J Med 2013, 368:2355-2365.

9. den Hertog HM, van der Worp HB, van Gemert HM, Algra A, Kappelle LJ, van Gijn J, Koudstaal PJ, Dippel DW, PAIS Investigators: Correction: PAIS paracetamol (acetaminophen) in stroke; protocol for a randomized, double blind clinical trial. BMC Cardiovasc Disord 2008, 8:29. ISCRTN74418480

10. Miller SW, Palesch YY: Comments regarding the recent OAST article. Stroke 2008, 39:e14.

11. Hofmeijer J, Kappelle LJ, Algra A, Amelink GJ, van GJ, van der Worp HB: Surgical decompression for space-occupying cerebral infarction (the Hemicraniectomy After Middle Cerebral Artery infarction with Life-threatening Edema Trial [HAMLET]): a multicentre, open, randomised trial. Lancet Neurol 2009, 8:326-333.

12. Ananth CV, Kleinbaum DG: Regression models for ordinal responses: a review of methods and applications. Int J Epidemio/ 1997, 26:1323-1333.

13. den Hertog HM, van der Worp HB, van Gemert HM, Algra A, Kappelle L, van Gijn J, Koudstaal PJ, Dippel DW, PAIS Investigators: The Paracetamol (Acetaminophen) In Stroke (PAIS) trial: a multicentre, randomised, placebo-controlled, phase III trial. Lancet Neurol 2009, 8:434-440.

14. Whitehead J: Sample size calculations for ordered categorical data. Stat Med 1993, 12:2257-2271.
15. Horan TC, Andrus M, Dudeck MA: CDC/NHSN surveillance definition of health care-associated infection and criteria for specific types of infections in the acute care setting. Am J Infect Control 2008, 36:309-332.

16. Katzan IL, Cebul RD, Husak SH, Dawson NV, Baker DW: The effect of pneumonia on mortality among patients hospitalized for acute stroke. Neurology 2003, 60:620-625.

17. Kwan J, Hand P: Infection after acute stroke is associated with poor short-term outcome. Acta Neurol Scand 2007, 115:331-338.

18. McHugh GS1, Butcher I, Steyerberg EW, Marmarou A, Lu J, Lingsma HF, Weir J, Maas Al, Murray GD: A simulation study evaluating approaches to the analysis of ordinal outcome data in randomized controlled trials in traumatic brain injury: results from the IMPACT project. Clin Trials 2010, 7:44-57.

19. Savitz SI, Lew R, Bluhmki E, Hacke W, Fisher M: Shift analysis versus dichotomization of the modified Rankin scale outcome scores in the NINDS and ECASS-II trials. Stroke 2007, 38:3205-3212.

doi:10.1186/1745-6215-15-133

Cite this article as: Westendorp et al:: Update on the Preventive Antibiotics in Stroke Study (PASS): a randomised controlled phase 3 clinical trial. Trials 2014 15:133.

\section{Submit your next manuscript to BioMed Central and take full advantage of:}

- Convenient online submission

- Thorough peer review

- No space constraints or color figure charges

- Immediate publication on acceptance

- Inclusion in PubMed, CAS, Scopus and Google Scholar

- Research which is freely available for redistribution

Submit your manuscript at www.biomedcentral.com/submit
C) Biomed Central 